\title{
Cerebral blood flow increases during insulin-induced hypoglycaemia in Type 1 (insulin-dependent) diabetic patients and control subjects
}

\author{
H.A.W.Neil ${ }^{1}$, E.A.M.Gale ${ }^{2}$, S.J.C.Hamilton ${ }^{4}$, I.Lopez-Espinoza ${ }^{2}$, R. Kaura ${ }^{3}$ and S.T.McCarthy ${ }^{4}$ \\ ${ }^{1}$ Department of Community Medicine and General Practice, University of Oxford and ${ }^{2}$ Sheikh Rashid Diabetetes Unit, \\ ${ }^{3}$ Nuffield Ophthalmology Laboratories and ${ }^{4}$ Department of Geriatric Medicine, The Radcliffe Infirmary, Oxford, OK
}

\begin{abstract}
Summary. The effect of insulin-induced hypoglycaemia on cerebral blood flow was examined using the intravenous xenon-clearance technique in 9 patients with Type 1 (insulindependent) diabetes (aged 20 to 43 years) and 9 age-matched control subjects before, during and after hypoglycaemia. Cerebral blood flow rose in both groups. The mean basal cerebral flood flow values were not significantly different and during hypoglycaemia mean cerebral blood flow increased by $17 \%(p=0.008)$ in the diabetic patients and by
\end{abstract}

$21 \%(p=0.0003)$ in the control subjects. The results suggest that in young diabetic patients without autonomic neuropathy or microangiopathy cerebral vessels dilate normally in response to hypoglycaemia. The physiological importance of an increase in cerebral blood flow during hypoglycaemia is uncertain; but glucose availability is increased.

Key words: Cerebral blood flow, hypoglycaemia, blood viscosity, Type 1 diabetes.
Hypoglycaemia is the most common complication of insulin therapy; about $10 \%$ of insulin-treated patients experience one or more episodes of severe hypoglycaemia annually [1-4]. Severe hypoglycaemia produces profound changes in the central nervous system; but surprisingly little is known about its effects on cerebral circulation in man.

Previous cross-cannulation studies using the nitrous oxide method to measure cerebral blood flow (CBF) during insulin-induced hypoglycaemia reported conflicting results. Eisenburg [5] and Kety [6] found no change in CBF but Porta [7] found a significant increase in schizophrenic patients treated by insulin shock therapy.

Our aim was to compare the effect of insulin-induced hypoglycaemia on CBF in young patients with Type 1 diabetes and age-matched normal control subjects.

\section{Subjects and methods}

CBF was studied in the two groups of subjects before, during and after insulin-induced hypoglycaemia. All subjects gave informed consent and the study was approved by the local ethics committee.

Group 1 . Nine healthy control subjects (median age: 31 years, range 25-37 years, six men, three women).

Group 2. Nine young Type 1 diabetic patients (median age: 30 years, range 20-43 years, eight men, one woman). All except one subject were free from microangiopathy: the clinical data for the group are shown in Table 1.

Retinopathy was assessed by direct ophthalmoscopy and an early morning urinary albumin concentration was measured by a double antibody immunoassay procedure [8]. The mean duration of diabetes ( $\pm 1 \mathrm{SD}$ ) was $11.5 \pm 6.5$ years, the mean insulin dose $/ \mathrm{kg}$ was $0.71 \pm$ $0.29 \mathrm{U} / \mathrm{kg}$ and the mean $\mathrm{HbA}_{1}$ was $11.2 \pm 2.9 \%$ (Corning method, upper limit of reference range $7.5 \%$ ).

None of the diabetic patients or control subjects had a history of head injury or cerebrovascular disease; electroencephalograms performed before the study were reported to be within normal limits. None of the diabetic patients had a history of severe recurrent hypoglycaemia but three subjects had experienced nocturnal hypoglycaemic reactions in the preceeding month and two had been unconscious once in the previous year. No drugs other than insulin were being taken at the time of the study and no subject had a body mass index of greater than 25.

Autonomic function was assessed using cardiovascular reflex tests (the standard deviation of the mean R-R interval over a 5-min period lying down, the expiratory to inspiratory ratio for a single deep breath, and the Valsalva ratio) and results outside the $97.5 \mathrm{cen}$ tile of age-related normal ranges [9-11] were defined as abnormal. The postural change in blood pressure was also measured. One diabetic patient with evidence of microangiopathy also had an abnormal expiratory to inspiratory ratio but all other subjects had normal autonomic function.

\section{Methods}

In diabetic patients short-acting insulin was substituted for intermediate and long-acting insulin $24 \mathrm{~h}$ before the study. Patients were admitted to hospital at 20.00 hours the evening before the study. Overnight normoglycaemia was achieved with a constant intravenous insulin infusion. Control subjects fasted overnight for a similar period but were not admitted. 
Table 1. Clinical data for Type 1 diabetic patients

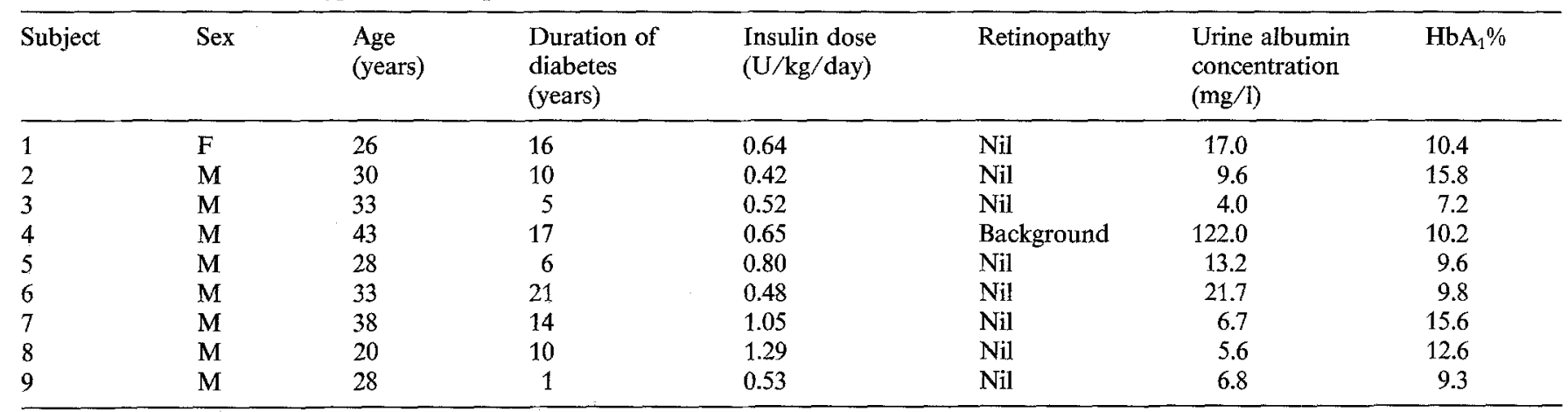

Table 2. Mean fasting, nadir and recovery glucose and basal haematocrit in diabetic patients and control subjects

\begin{tabular}{lcc}
\hline & $\begin{array}{l}\text { Diabetic } \\
\text { patients } \\
(n=9)\end{array}$ & $\begin{array}{l}\text { Control } \\
\text { subjects } \\
(n=9)\end{array}$ \\
\hline Fasting glucose (mmol/1) & $5.3 \pm 1.3$ & $4.7 \pm 0.6$ \\
Nadir glucose & $1.3 \pm 0.5$ & $1.1 \pm 0.6$ \\
Recovery glucose & $8.8 \pm 2.7$ & $6.8 \pm 1.3$ \\
Time to hypoglycaemia (min) & $54 \pm 13$ & $43 \pm 12$ \\
Basal haematocrit & $0.425 \pm 0.025$ & $0.430 \pm 0.035$ \\
\hline
\end{tabular}

Results are stated as mean $\pm 1 \mathrm{SD}$

The study began at 08.00 hours with a basal measurement of CBF using a non-invasive intravenous xenon ${ }^{133}$ clearance technique [12]. Five to seven millicuries of xenon were given by bolus injection into a forearm vein which was then flushed with isotonic saline while subjects held their breath for $10 \mathrm{~s}$. A minimal peak count of 20,000 counts per $5 \mathrm{~s}$ was ensured. Two opposed $3.8 \mathrm{~cm}$ diameter, $3 \mathrm{~mm}$ thick, well-screened sodium iodide scintillation detectors, with $5 \mathrm{~cm}$ parallel whole collimators were used. The counters were placed with the posterior edge of each collimator just in front of the parietal eminence. The detectors were set symmetrically $(57-105 \mathrm{Kev})$ around the $81 \mathrm{Kev}$ gamma peak of xenon ${ }^{133}$. A 5-s sampling interval was used and the clearance of the isotope was measured for $12 \mathrm{~min}$. Air samples were drawn from a face mask into a separate sodium iodide air detector throughout the procedure to measure the activity in the expired air. The data were collected onto floppy disk using a $\mathrm{Nu}$ clear Data 66 computer and the clearance curves were analysed later on a DEC $11 / 34$ computer by a modification of the method described by Obrist [13]. To avoid extra-cerebral contamination only the fast clearing time curves, corresponding mainly to grey matter, were used. End tidal $\mathrm{CO}_{2}$ was monitored using a Beckman infra-red $\mathrm{CO}_{2}$ analyser and chart recorder. Blood pressure was recorded with an electronic sphygmomanometer (Opal UA-251) and the mean of 3 readings was used. The ECG was continuously displayed and the pulse was monitored.

Blood specimens were taken via an indwelling intravenous cannula in an antecutibal vein for measurement of glucose, $\mathrm{HbA}_{1}$, full blood count, platelets and fibrinogen. Plasma glucose was measured by the glucose oxidase method. Specimens were also taken in seven diabetic patients and four control subjects for determination of whole blood viscosity which was measured within $1 \mathrm{~h}$ at $37^{\circ} \mathrm{C}$ in a Couette type cup and bob viscometer (Contraves Low Shear 30). The shear rate was increased logarithmically from 0 to $128.5 / \mathrm{s}$ with a ramp time of $1 \mathrm{~min}$. Low shear rate readings for whole blood viscosity were taken at the lowest recordable shear rate so that differences between individuals, due to the non-Newtonian behaviour of blood, could be detected before the rheological properties were altered at higher viscosities. Standardised low shear rate readings were also taken at a rate of $0.277 / \mathrm{s}$ and high shear readings at $94.5 / \mathrm{s}$.
An intravenous infusion of Velosulin insulin at a rate of 0.15 to $0.20 \mathrm{U} \cdot \mathrm{kg}^{-1} \cdot \mathbf{h}^{-1}$ was used to induce hypoglycaemia which was defined as a glucose of less than $2.0 \mathrm{mmol} / 1$ together with symptoms and signs of hypoglycaemia. At this point CBF and other measurements were repeated and the insulin infusion was continued until the CBF measurement was complete. Hypoglycaemia was then reversed with oral glucose. An hour later, the final recovery phase CBF measurement was made.

\section{Statistical analysis}

Paired Student's t-tests were used for comparison of data within groups and unpaired t-tests for comparison between groups. Correlations were assessed using Pearson's correlation coefficient. 95\% confidence intervals were calculated for the difference between means. Two-tailed tests of significance were reported and a value of $p<0.05$ was considered statistically significant. The results are stated as the mean $\pm 1 S D$.

\section{Results}

Table 2 shows the mean fasting, nadir and recovery glucose in diabetic patients and control subjects. The nadir glucose was $1.3 \pm 0.5 \mathrm{mmol} / 1$ in diabetic patients and $1.1 \pm 0.6 \mathrm{mmol} / 1$ in control subjects. There was no significant difference between either the mean fasting or nadir glucose in the two groups.

The systolic blood pressure increased significantly and the diastolic fell significantly during hypoglycaemia in patients and controls (Table 3). There was a corresponding fall in mean arterial pressure (systolic $\mathrm{BP}+2 \times$ diastolic $\mathrm{BP} / 3$ ) in both groups, a small but insignificant rise in the platelet count and no change in plasma fibrinogen.

The mean basal CBF was almost identical in the 2 groups and increased significantly during hypoglycaemia CBF (Table 4): the mean increase was $16.7 \pm$ $14.5 \%$ in patients and $21.1 \pm 10.1 \%$ in controls. After reversal of hypoglycaemia, the mean CBF remained elevated (recovery phase CBF results were unavailable for one control subject). Cerebrovascular resistance (mean arterial blood pressure/CBF) fell by $16.1 \pm$ $12.6 \%$ in patients $(p=0.009)$ and $22.5 \pm 10.6 \%$ in controls $(p=0.008)$ during hypoglycaemia. The maximum absolute change in end tidal $\mathrm{CO}_{2}$ in any subject was a 
Table 3. Mean arterial, systolic and diastolic blood pressures in diabetic patients and control subjects before, during and after hypoglycaemia

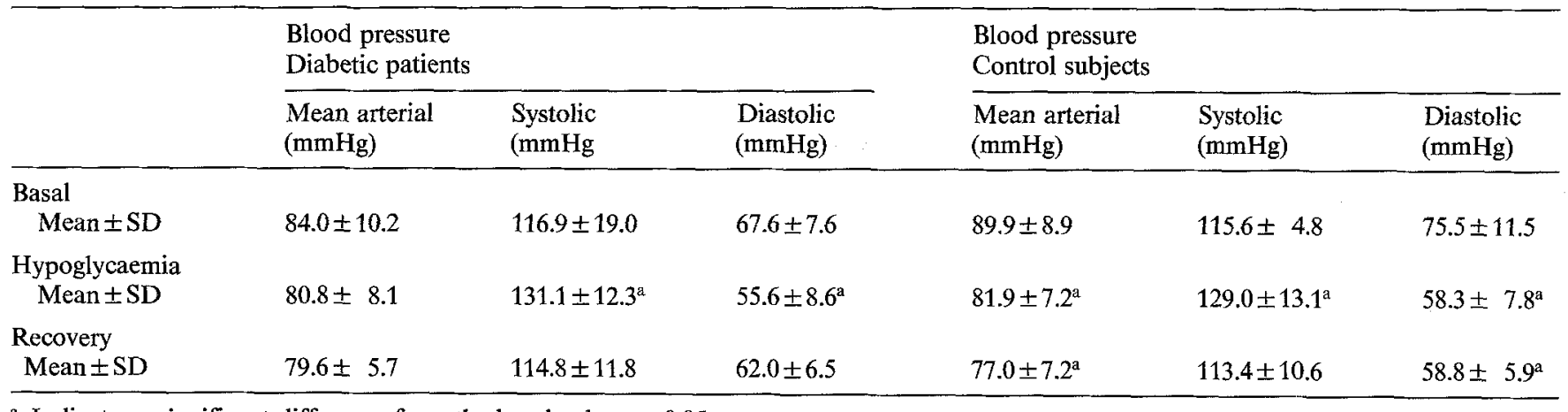

a Indicates a significant difference from the basal value $p<0.05$

Table 4. CBF in diabetic patients and control subjects before, during and after hypoglycaemia

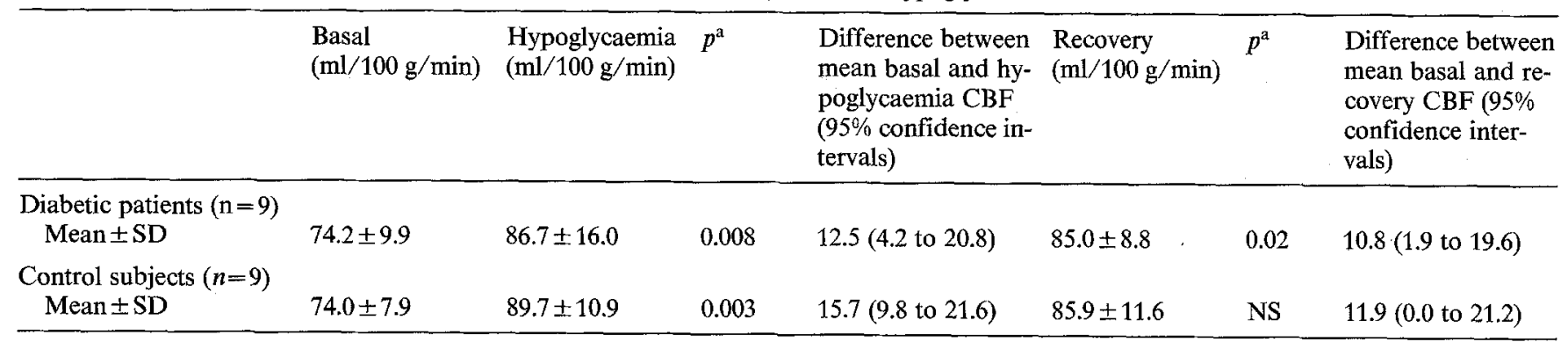

a Significance of difference from basal value

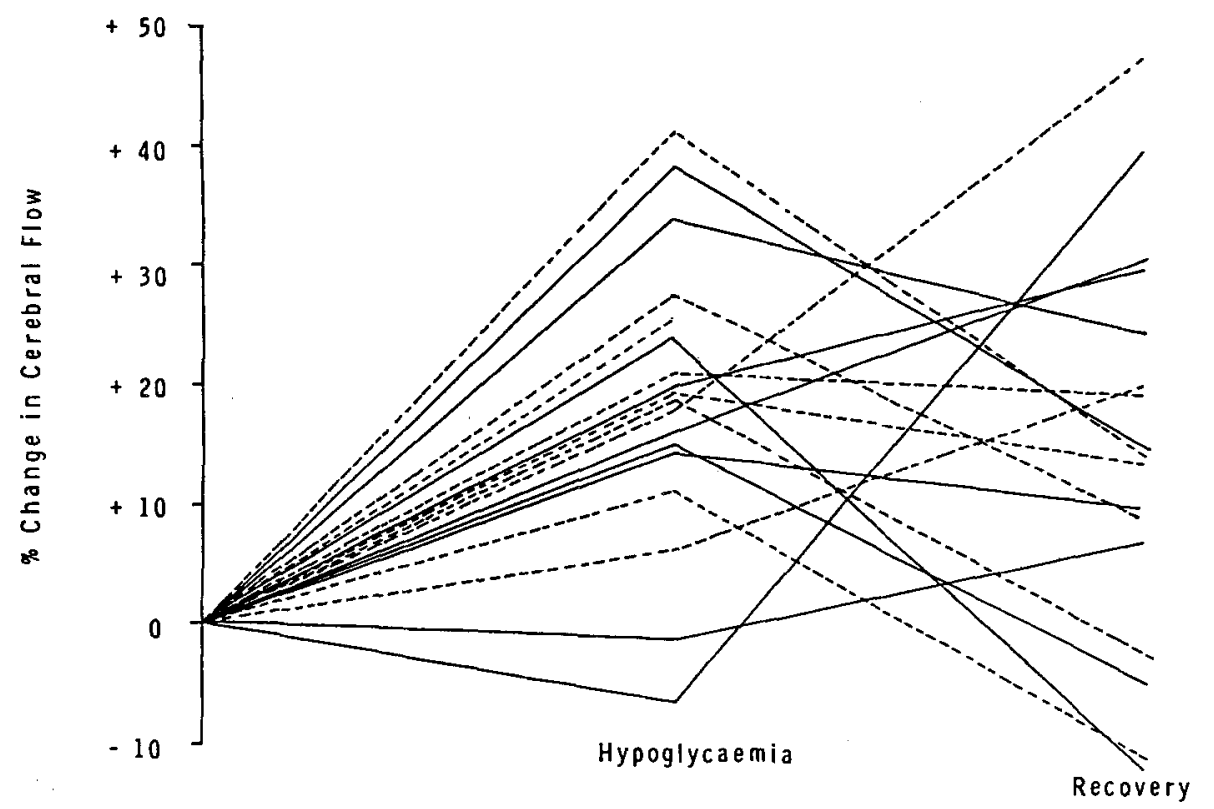

Fig. 1. Percentage change in CBF during hypoglycaemia and after its reversal in 9 Type I diabetic patients (-) and 9 agematched control subjects (-----). reduction of $0.5 \%$. Figure 1 shows graphically the percentage change in $\mathrm{CBF}$ from the basal measurement. In 11 subjects the maximum increase occurred during hypoglycaemia but in 2 control subjects and in 4 patients it was delayed until after its reversal. CBF actually fell during hypoglycaemia in one patient and subsequently increased during the recovery phase. The maximum increase in CBF (during hypoglycaemia or after recovery) is shown in Table 5: the mean increase was $25.7 \pm 11.6 \%$ in patients and $26.7 \pm 12.3 \%$ in con- trol subjects. There was no significant correlation between fasting glucose values and CBF, nor between changes in glucose and changes in $\mathrm{CBF}$, during either hypoglycaemia or after its reversal.

High and low shear rate whole blood viscosity in creased during hypoglycaemia and returned to basal levels after its reversal. There was no significant correlation between high shear rate viscosity and CBF during hypoglycaemia $(r=0.50)$. In contrast, there was a significant negative correlation of $-0.77(p=0.006)$ be- 
Table 5. Maximum CBF in diabetic patients and control subjects (during hypoglycaemia or in the recovery phase)

\begin{tabular}{llll}
$\begin{array}{l}\text { Basal } \\
(\mathrm{ml} / 100 \mathrm{~g} /\end{array}$ & $\begin{array}{l}\text { Maximum } \\
(\mathrm{ml} / 100 \mathrm{~g} /\end{array}$ & $p$ & $\begin{array}{l}\text { Difference be- } \\
\text { tween means } \\
\mathrm{min})\end{array}$ \\
& $\begin{array}{l}\text { min) } \\
\end{array}$ & $\begin{array}{l}95 \% \text { confi- } \\
\text { dence inter- } \\
\text { vals })\end{array}$ \\
\hline
\end{tabular}

Diabetic

patients

$(n=9)$

Mean \pm SD $\quad 74.2 \pm 9.9 \quad 92.6 \pm 9.7 \quad 0.0001 \quad 18.4$

(12.7 to 24.2$)$

Control subjects

$(n=9)$

Mean \pm SD $\quad 74.0 \pm 7.9 \quad 93.5 \pm 10.8<0.0001 \quad 19.5(12.9$ to

26.1)

tween low shear rate viscosity and $\mathrm{CBF}$ and the correlation was unchanged using either the lowest recordable shear rate or a standardised rate of $0.277 / \mathrm{s}$. There was also a significant correlation of $0.87(p=0.001)$ between cerebrovascular resistance and the standardised low shear rate viscosity during hypoglycaemia.

\section{Discussion}

Earlier studies of CBF during insulin-induced hypoglycaemia have reported conflicting results. Kety and Schmidt [6] used the nitrous oxide method to study CBF in 7 schizophrenic patients during insulin shock therapy. Results were available for only 4 patients during hypoglycaemia and 3 patients during coma; no significant change in $\mathrm{CBF}$ was observed. Eisenburg and Seltzer [5] also used the nitrous oxide method to study 16 subjects and reported an increase in CBF in 10 subjects although the overall increase was not significant. However, comparison between their results and our own is complicated by the significantly higher mean nadir glucose in their study.

We found a significant increase in CBF during severe hypoglycaemia in young diabetic patients with Type 1 diabetes and age-matched control subjects. This confirms the findings of Porta et al. [7] and extends them to young Type 1 diabetic patients without microangiopathy. By contrast, in a group of diabetic patients with a higher mean age of 46 years (range 14-76), Dandona et al. [14] showed that the reactivity of cerebral blood vessels to carbon dioxide was altered. They found CBF was increased in control subjects but was unchanged or reduced in many patients including newly-diagnosed diabetic patients without complications. They suggested that this might be caused by some form of neurohumoral dysregulation or alteration in receptors on vascular smooth muscle and that the impaired ability of cerebral vessels to dilate was a complication of diabetes. In long-term Type 1 diabetic patients with retinopathy and nephropathy, Kastrup et al. [15] have also shown that autoregulation of $\mathrm{CBF}$ is impaired.
During hypoglycaemia total peripheral resistance decreases [16] and we found a corresponding decrease in cerebrovascular resistance. However, the stimulus for cerebral vasodilatation remains unclear. In the peripheral circulation there is evidence that the increase in blood flow to the extremities is mostly due to withdrawal of vasoconstrictor tone [17] but the evidence for a neurogenic role in cerebral autoregulation is less convincing. Case studies of patients with autonomic neuropathy due to the Shy-Drager syndrome [18-21] indicate that cerebral vascular reactivity to $\mathrm{CO}_{2}$ is preserved; but both normal and impaired autoregulation have been reported. The effect of diabetic autonomic neuropathy on cerebral autoregulation is therefore uncertain. In our study only one patient had any evidence of autonomic cardiovascular dysfunction and he failed to show a significant rise in CBF.

Arterial oxygen content is the major determinant of CBF [22-23] and an increased respiratory rate during hypoglycaemia may have influenced CBF. However, in 6 subjects who had a raised low shear rate viscosity during hypoglycaemia we found that the maximum increase in CBF was delayed until after the reversal of hypoglycaemia. In these subjects, it is likely that a raised haematocrit was the principal component of the increased viscosity [24]. In turn, this increases the oxygen-carrying capacity of the blood and CBF either fails to rise, or even falls, so that a constant brain oxygen tension is preserved. This is also consistent with a significant fall in plasma volume and an increase in haematocrit reported in some normal subjects during insulin-induced hypoglycaemia by Hilsted et al. [16]. There are similarities between our findings and observations by Porta et al. [7] of a fall in CBF in 8 of 14 subjects 90 min after an intramuscular injection of insulin. This was followed by a $38 \%$ rise in mean CBF after 4 to $5 \mathrm{~h}$ coma. In 3 subjects, the maximum increase in CBF was delayed until the reversal of hypoglycaemia.

The metabolic significance of increased CBF during severe hypoglycaemia is uncertain. In rats, CBF increases by $200 \%$ and autoregulation is lost during experimental hypoglycaemia of sufficient severity to cause cessation of spontaneous EEG activity. Nerve cell damage occurs in areas which show a reduced cerebral metabolic rate for glucose during hypoglycaemia and reduced regional blood flow during the recovery period [25]. In man, during less severe hypoglycaemia, raised $\mathrm{CBF}$ may compensate for a reduction in plasma glucose by increasing glucose availability. Increasing linear flow rates results in only a small increase in glucose transport across the blood brain barrier because this is diffusion limited. However, an increase in flow by recruitment of capillaries increases the capillary area and may correspondingly increase glucose transport [26].

In summary, we found a significant increase in CBF during severe hypoglycaemia both in young 
Type 1 diabetic patients and in control subjects. The results suggest that in young diabetic patients without autonomic neuropathy or microangiopathy cerebral vessels dilate normally in response to hypoglycaemia. The metabolic significance of a rise in $\mathrm{CBF}$ during hypoglycaemia is unclear but glucose availability is increased, although there is not necessarily a parallel increase in glucose oxidation. Further evidence is needed to determine whether the neurological risks of severe hypoglycaemia are increased if this response is delayed or absent.

Acknowledgements. We are grateful to Dr. T.D.R. Hockaday for advice and helpful criticism, to Dr. E. Poole and the staff of his department for undertaking the EEGs, to Mr R.F.Smith for glucose measurements and to Ms J.McClean-Bushnell for microalbumin measurements.

\section{References}

1. Potter J, Clarke P, Gale EAM, Dave SH, Tattersall RB (1982) Insulin-induced hypoglycaemia in an accident and emergency department: the tip of an iceberg? Br Med J 285: 1180-82

2. Barnett AH, Leslie D, Watkins PJ (1980) Can insulin-treated diabetics be given beta-adrenergic blocking drugs? $\mathrm{Br}$ Med $\mathrm{J} \mathrm{I}$ : 976-978

3. Goldgewicht C, Slama G, Papoz L, Tchobroutsky G (1983) Hypoglycaemic reactions in 172 Type 1 (insulin-dependent) diabetic patients. Diabetologia 24: 95-99

4. Mühlhauser I, Berger M, Sonnenberg G, Koch J, Jörgens V, Schernthaner G, Scholz V (1985) Incidence and management of severe hypoglycaemia in 434 adults with insulin-dependent diabetes mellitus. Diabetes Care 8: 268-73

5. Eisenburg S, Seltzer HS (1962) The cerebral metabolic effects of acutely induced hypoglycemia in human subjects. Metabolism 11: $1162-1168$

6. Kety SS, Schmidt CF (1948) Cerebral blood flow and metabolism in schizophrenia. The effects of barbiturate semi-narcosis, insulin and electroshock. Am J Psychiat 104: 765-770

7. Porta PD, Maiolo AT, Negri VU, Rossella E (1964) Cerebral blood flow and metabolism in therapeutic insulin coma. Metabolism 13: 131-140

8. Samuell CT, Walker BJ, Smith RF, Dhor H, Nelstrop GA (1984) Assay of microalbuminuria using gel electroimmunoassay. Diabetic Medicine 1: 298-300

9. Smith SA (1984) Diagnostic value of the valsalva ratio reduction in diabetic autonomic neuropathy: use of an age-related normal range. Diabetic Medicine 1: 295-7

10. Smith SA (1982) Reduced sinus arrhythmia in diabetic autonomic neuropathy: diagnostic value of an age-related range. Br Med J 285: 1599-1601

11. Smith SE, Smith SA (1981) Heart rate variability in healthy subjects measured with a bedside computer-based technique. Clin Sci $61: 379-83$
12. Thomas DJ, Zilkha E, Redmond S, Du Boulay GH, Marshall J, Ross Russell, Symon L (1979) An intravenous ${ }^{133}$ xenon clearance technique for measuring cerebral blood flow. Neurol Sci 40: 53-63

13. Obrist WD, Thompson HK, Wang WS, Wilkingson WE (1975) Regional blood flow estimated by ${ }^{133}$ xenon inhalation. Stroke 6: 245-256

14. Dandona P, James IM, Newbury PA, Woollard ML, Beckett AG (1978) Cerebral blood flow in diabetes mellitus: evidence of abnormal cerebrovascular reactivity. Br Med J 11: 325-326

15. Kastrup J, Rorsgaard S, Parving HH, Lassen NA (1986) Impaired autoregulation of cerebral blood flow in long-term type I (insulin-dependent) diabetic patients with nephropathy and retinopathy. Clin Physiol 6: 549-59

16. Hilsted J, Bonde-Petersen F, Noorgaard M-B, Greniman M, Christensen NJ, Parving HH, Suzuki M (1984) Haemodynamic changes in insulin-induced hypoglycaemia in normal man. Diabetologia 26: $328-32$

17. Allwood MJ, Ginsburg J, Paton A (1957) The effect of insulin hypoglycaemia on blood flow in intact and sympathectomised extremities in man. J Physiol 139: 97-107

18. Meyer JS, Shirmazu K, Fukuuchi Y, Ohuchi T, Okamoto S, Koto A, Ericsson AD (1973) Cerebral dysautoregulation in central neurogenic orthostatic hypotension (Shy-Drager syndrome). Neurology (Minneap) 23: 262-73

19. Gotah F, Ebihara SI, Toyoda M, Shinohara Y (1971) Role of autonomic nervous system in human cerebral circulation. In: Fierschi C (ed) Proceedings of Fifth International Symposium on Cerebral Blood Flow and intracranial Pressure. Roma-Siena. Europ Neurol 6: 203-7

20. Carrona JJ, Plum F (1973) Cerebrovascular regulation in preganglionic and postganglionic autonomic insufficiency. Stroke 4: $12-19$

21. Depresseux JC, Rousseau JJ, Frank G (1979) The autoregulation of cerebral blood flow, the cerebrovascular reactivity and their interaction in the Shy-Drager Syndrome. Eur Neurol 18: 295-301

22. Brown MM, Marshall J (1985) Regulation of cerebral blood flow in response to changes in blood viscosity. Lancet 1:604-609

23. Brown MM, Wade JPH, Marshall J (1985) Fundamental importance of arterial oxygen content in regulation of cerebral blood flow in man. Brain 108: 81-93

24. Begg TB, Hearns JB (1966) Components in blood viscosity. Clin Sci 31: 87-93

25. Abdul-Rahman A, Siesjö BK (1980) Local cerebral glucose consumption during insulin-induced hypoglycaemia, and in the re covery period following glucose administration. Acta Physiol Scand 110: 149-159

26. Lund-Andersen $H$ (1979) Transport of glucose from blood to brain. Physiol Rev 59: 305-352

Received: 30 October 1986

and in revised form: 2 April 1987

Dr. H.A.W. Neil

Department of Medicine

Floor 4-Clinical Block

The Medical School

Framlington Place

Newcastle upon Tyne NE2 4HH

UK 